\title{
A direct plasma assay of circulating microRNA-210 of hypoxia can identify early systemic metastasis recurrence in melanoma patients
}

\author{
Shigeshi Ono${ }^{1}$, Takashi Oyama ${ }^{1}$, Stella Lam $^{1}$, Kelly Chong${ }^{1}$, Leland J. Foshag ${ }^{2}$, \\ Dave S.B. Hoon ${ }^{1}$ \\ ${ }^{1}$ Department of Molecular Oncology, John Wayne Cancer Institute, Providence Saint John's Health Center, Santa Monica, CA, USA \\ ${ }^{2}$ Division of Surgical Oncology, John Wayne Cancer Institute, Providence Saint John's Health Center, Santa Monica, CA, USA \\ Correspondence to: \\ Dave S.B. Hoon, e-mail: hoond@jwci.org \\ Keywords: cell-free microRNA, diagnosis, plasma, metastatic melanoma recurrence, LDH \\ Received: January 06, $2015 \quad$ Accepted: January 10, $2015 \quad$ Published: February 05, 2015
}

\section{ABSTRACT}

Circulating cell-free(cf) microRNAs (miRNAs) have been reported to exist in plasma. MicroRNA-210(miR-210) is known to play important roles in the tumor hypoxic state. We hypothesized that the expression levels of cf-miR-210 in plasma would predict early clinical recurrence in melanoma patients. A direct miRNA assay on plasma (RT-qPCR-DP) was developed to improve cf-miRNA assay logistics, eliminate RNA extraction, and reduce specimen amount required. RNA was extracted from formalin-fixed paraffin-embedded (FFPE) melanoma tissues $(n=108)$ and assessed by RT-qPCR. Plasma ( $10 \mu \mathrm{l} ; n=264$ ) was procured from AJCC Stage III/IV patients in phase III clinical trials. A RT-qPCR-DP was performed to detect cf-miR-210. MiR$\mathbf{2 1 0}$ was significantly higher in metastatic tumors compared to primary tumors. CfmiR-210 was significantly higher in melanoma patients versus healthy donor controls. In serial bloods within individual patients, cf-miR-210 $<3$ months prior to disease recurrence significantly increased compared to baseline levels $(p=0.012)$. ROC curve analysis demonstrated that patients with elevated cf-miR-210 were more likely to have disease recurrence. Moreover, cf-miR-210 increase significantly correlated with poorer prognosis $(p<0.001)$. Lactate dehydrogenase $($ LDH $)$ level was also assessed within patients, and the AIC values for proportional hazards regression models of cf-miR-210(120.01) and LDH (122.91) demonstrated that cf-miR-210 is a better recurrence indicator. We concluded enhanced cf-miR-210 provides identification of early systemic melanoma recurrence.

\section{INTRODUCTION}

Despite significant advances in new FDA approved drugs for malignant cutaneous melanoma, the 5-year survival of patients with American Joint Committee on Cancer (AJCC) stage IV disease is poor. AJCC stage III patients who undergo surgical resection of metastatic lymph nodes (LN) have better outcome especially when performed early after positive sentinel LN biopsy $[1,2]$. To better predict systemic recurrence after the resection of regional disease, reliable blood biomarkers are highly needed. Currently the only AJCC approved blood biomarker is lactate dehydrogenase (LDH) which has been used as an indicator and progression factor in metastatic stage IV disease in melanoma for several decades. However, LDH has a limitation in accuracy and early systemic recurrence. As reported in the literature most blood cancer biomarkers predictive for systemic recurrence have not been shown to provide an accurate early time frame prior to actual clinical recurrence. We are in need of better and more efficient blood biomarkers for early systemic melanoma recurrence. This is highly important for metastatic melanomas where they are often highly aggressive in spreading and fatal. Earlier detection of systemic disease may provide an advantage for treatment such as surgery in particular recently [2], in 
light of new therapeutic drug regimens approved by the FDA for melanoma in the last 3 years [3, 4].

John Wayne Cancer Institute initiated prospective clinical phase III multicenter studies (the Malignant Melanoma Active Immunotherapy trials; MMAIT) in patients with AJCC stage III and IV melanoma independently to assess the efficacy of adjuvant treatment with active-specific immunotherapy. These were FDA phase III, randomized clinical trials, and patients were regularly followed up for $>5$ years as defined in the clinical protocol. Patients had prospective serial bloods drawn on a defined schedule at specific intervals under Good Laboratory Practice (GLP) conditions $[5,6]$. The patients were all determined to be rendered clinically disease-free before entering the trials based upon clinical assessment $[5,6]$. This unique patient resource has allowed us to assess possible blood biomarkers and their utility prior to clinical evidence of recurrence. In most studies blood cancer biomarkers are assessed in patients with clinical disease present which complicates interpretation of the biomarker efficacy.

MicroRNAs (miRNAs) are small single-stranded noncoding RNA molecules that interact with their target mRNAs to inhibit translation by degrading mRNA or to block translation without degrading the mRNA by binding to complementary sequences in the 3 ' untranslated regions (3' UTR) of mRNA [7-9]. The advantage of assessing miRNAs in blood as biomarkers is that they are more stable at room temperature compared to mRNA [10], can sustain multiple freeze-thaw cycles, and survive the effects of RNase and DNase whereby mRNA degrades rapidly [11]. The remarkable stability of miRNAs in blood makes it a suitable biomarker. Expression profiles of serum/plasma miRNAs can discriminate patients with specific cancers [11-13]. While several studies have demonstrated cell-free(cf)-miRNAs potential as a biomarker in melanoma patients $[14,15]$, none of them has been clinically utilized or has accurately predicted recurrence in a well-defined phase III clinical trial setting patients with accurate clinical documentation and blood draws during follow-up.

We previously established a direct qPCR assay to study circulating DNA in serum from patients with breast and other cancers $[16,17]$. More recently we established a RT-qPCR directly-in-serum (RT-qPCR-DS) assay for breast cancer where the RT is directly performed in serum without the need for RNA extraction [18]. Efficient and reproducible extraction of circulating cf-nucleic acids from blood has been problematic, particularly when the available amount of nucleic acids or source material is limited in quantity. The "Achilles heel" of cf-nucleic acid detection has been the extraction from body fluids, and logistical approaches of assessing miRNA have additional problems because of their small size. RT-qPCR-DS is logically simpler and more responsive in assessing cf-miRNAs than extracting RNA, as it eliminates the inevitable loss of miRNAs during the extraction step. In the current study, we applied a modified RT-qPCR directly-in-plasma assay (RT-qPCR-DP) to detect cfmiRNAs in low volume of plasma.

Hypoxia is known to be a common characteristic of the tumor microenvironment in advanced solid cancers, especially rapidly growing tumors due to inadequate blood supply in the central area of the tumor $[19,20]$. MicroRNA-210 (miR-210) is one of the most significantly upregulated miRNAs in a hypoxic state, and has been reported to be an oncogenic miRNA [21-24]. It has also been shown to promote stem cell survival by targeting CASPSAP2 [25], and to stall DNA repair by targeting RAD52 [26], which are beneficial for cancer progression. We hypothesized that the expression levels of cf-miR-210 could be correlated with melanoma metastasis and be used for early identification of systemic disease recurrence.

Our studies demonstrated the utility of a direct plasma assay to detect cf-miRNA, potential of monitoring early events of clinical tumor hypoxia, and use of miR-210 in cutaneous melanoma patients to identify systemic recurrence early.

\section{RESULTS}

\section{MiR-210 expression level in metastatic melanoma compared to primary tumor}

RT-qPCR analysis of total RNA extracted from formalin-fixed paraffin-embedded (FFPE) melanomas confirmed miR-210 expression level was significantly higher in both lymph node metastasis (LNM) and distant organ metastasis (DOM) compared to primary tumors (PRM; Figure 1A; $p=0.002,<0.001$, respectively). Eight PRMs had paired metastases from DOM whereby we could demonstrate miR-210 level was significantly higher in DOM compared to respective PRM in all eight pairs (Figure 1B; $p<0.001$ ). This demonstrated the development of the hypoxic miR-210 relevance to melanoma metastasis occurrence.

\section{Analytical sensitivity, specificity, and reproducibility of RT-qPCR-DP}

We generated a standard curve using a serial dilution of melanoma cell line (M14) for efficiency and level of detection in the PCR assays. All plasma samples were assessed in triplicate $\mathrm{PCR}$ reaction. The $\mathrm{SD}$ of $\mathrm{dCq}$ values for three individual plasma samples ranged from 0.24 to 0.93 with a mean of 0.79 . The samples assessed in separate RT-qPCR-DP assays produced consistent dCq values. Run 1: dCq values were (A) 5.00, (B) 7.70, and (C) 5.19. Run 2: dCq values at (A) 4.01, (B) 7.40, and (C) 5.69. Run 3: dCq values were (A) 5.32, (B) 7.62, and 
(A)

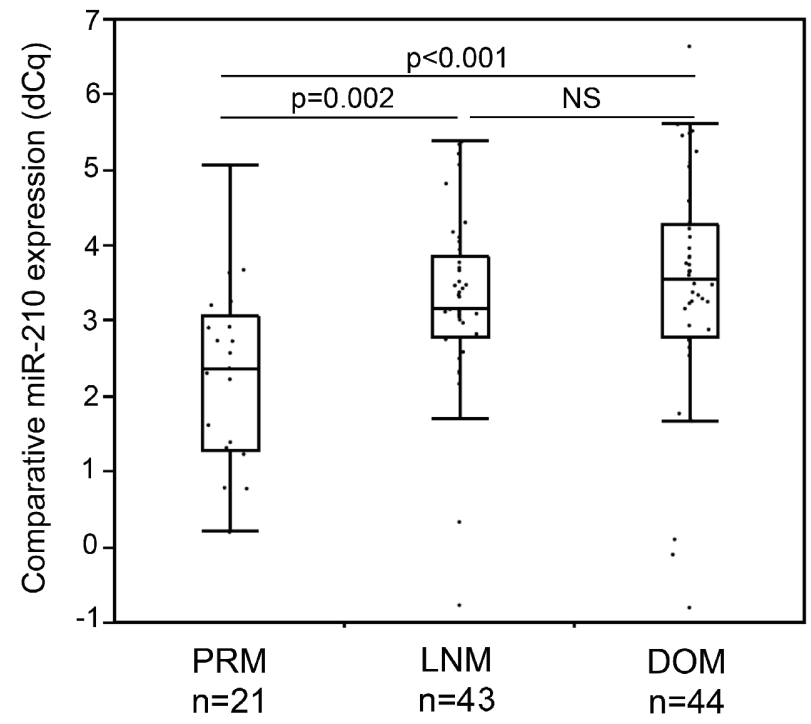

(B)

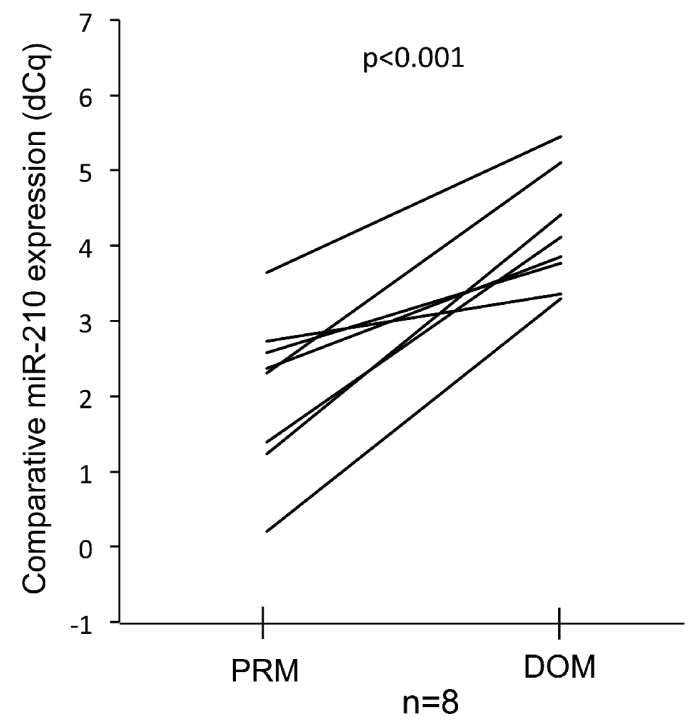

Figure 1: Comparison of miR-210 expression in melanoma FFPE samples. (A) MiR-210 expression level was significantly higher in LNM or DOM melanoma samples compared to that in PRM ( $p=0.002,<0.001$, respectively). (B) In paired samples in the same patients, DOM had significantly higher miR-210 level than PRM $(p<0.001)$.

(C) 6.48 with the SDs of (A) 0.68, (B) 0.16 and (C) 0.65 . This indicated RT-qPCR-DP could detect cf-miR-210 with high reproducibility.

A comparative assessment between with or without the Centri-Sep purification in the assay $(n=9$; healthy donors: $n=5$, stage IV: $n=4$ ) indicated a significant increase in sensitivity $(p<0.001)$ with purification (Supplemental Figure 1).

\section{Cf-miR-210 expression in melanoma patients with disease present: pilot study}

We then performed a pilot study to assess plasma cfmiR-210 expression in melanoma patients with different levels of metastatic disease present compared to healthy donor controls (healthy donors: $n=6$; stage III: $n=20$; stage IV: $n=26$ ). As shown in Figure 2, cf-miR-210 could be detected by RT-qPCR-DP, and the expression level significantly increased with higher AJCC stage.

\section{Cf-miR-210 in disease-free patients compared to healthy donors: verification study}

Utilizing a patient cohort A comprising of 130 AJCC Stage III $(n=60)$ and IV $(n=70)$ melanoma patients, cf-miR-210 detection in plasma was assessed by RTqPCR-DP and compared between the disease-free melanoma patients and 35 healthy donors. The bleeding times of patients were selected based on availability. As shown in Figure 3, the cf-miR-210 level was significantly higher in the disease-free patients compared to healthy donors $(p<0.001)$.

\section{Cf-miR-210 expression increase before disease recurrence}

Using another patient group, cohort B, comprising of AJCC stage III patients $(n=88)$ from the MMAIT-III, we compared cf-miR-210 detection in plasma taken before adjuvant treatment after being rendered disease free by surgery as the baseline, to their detection in paired serial bleeds procured from the same patient, respectively.

Cf-miR-210 expression in disease recurrent patients significantly increased prior to clinical recurrence compared to the baseline level $(p=0.012)$, however, no significant change in cf-miR-210 occurred in nonrecurrent patients (Figure 4A, 4B).

No correlation was detected between the baseline cfmiR-210 expression and the length of time from surgery to the bleeding point. There was also no correlation between cf-miR-210 expression and gender or age. In addition, the type of adjuvant treatment the patient underwent $(\mathrm{BCG}+$ placebo or BCG + Canvaxin $^{\circledR}$ ) was not associated with patients' outcome or cf-miR-210 expression.

\section{Stage III patients with elevated cf-miR-210 before systemic disease recurrence correlated to poor prognosis}

In the patient cohort $\mathrm{B}$, patients whose cf-miR-210 expression increased $>1 \mathrm{dCq}$ from the baseline to their paired plasma were significantly more likely to recur. Receiver-operating characteristics (ROC) curve analysis was performed, and AUC was 0.623 ( $p=0.015$; Figure 5A). The Stage III patients with enhanced 


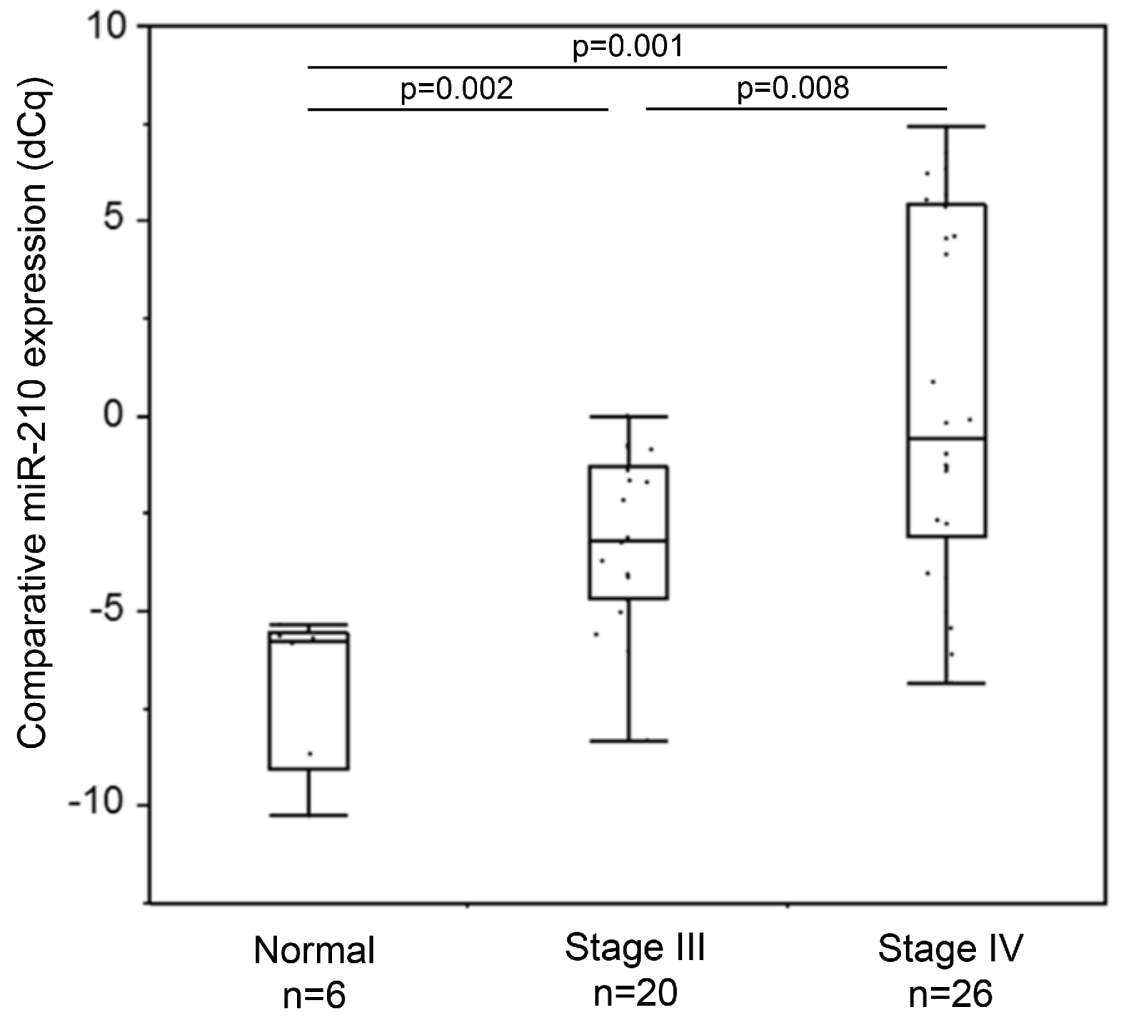

Figure 2: Cf-miR-210 expression in healthy donors and melanoma patients with clinical disease. Cf-miR-210 level increased according to AJCC stage progression.

cf-miR-210 expression also experienced significantly poorer prognosis compared to patients whose cf-miR-210 expression did not significantly change. The differences in disease-free survival (DFS) and melanoma-specific survival (MSS) were highly significant (DFS: $p<0.001$; MSS: $p<0.001$; Figure 5B). An increase in cf-miR-210 expression in plasma was not associated with the adjuvant treatment type the patient received.

\section{Cf-miR-210 compared to lactate dehydrogenase (LDH)}

LDH levels of the recurrent patients in blood were also analyzed. It was from either the same day or $<30$ days of the plasma for which cf-miR-210 was assessed. Although elevated LDH showed a trend for predicting disease recurrence, it was not significant $(p=0.081$; Figure 6A). However, an increase in cf-miR-210 was a significant predictor of disease recurrence $(p=0.015$; Figure 6B). Akaike information criterion (AIC) values for proportional hazards regression models of cf-miR-210 was lower than that of LDH (AIC $=120.01,122.91$, respectively), which demonstrated cf-miR-210 was the better indicator of disease recurrence. Combination of cf-miR-210 and LDH did not significantly improve performance for prediction of melanoma recurrence $(\mathrm{AIC}=120.03)$.

\section{DISCUSSION}

There are several tissue-based, blood-based and in vitro functional studies of miRNAs in melanoma [14, 27-31], however, the role of hypoxic miR-210 in melanoma remains unclear. In addition, there has been no significant report about cf-miR-210 in melanoma related to monitoring disease outcome.

MiR-210 is the most eminent hypoxia-inducible miRNA that is over-expressed in several cancers [24]. Rapidly proliferating tumors may outgrow their blood supply giving rise to hypoxia within the tumor that causes over-expression of miR-210 for angiogenesis [19, 20, 32]. In addition, several reports have indicated that miR-210 is correlated with a poor prognosis in various cancers [24]. In this study, we described the efficacy of measuring cfmiR-210 in plasma as a prognostic biomarker for early systemic disease recurrence for the first time.

We confirmed that the level of miR-210 in melanomas tissues was significantly higher in metastases compared to PRMs. There was also a significant difference between the paired-PRM and DOM melanomas within patients. These results implied miR-210 was elevated during systemic disease progression. It has been also reported that hypoxia is related to disease progression through vascular endothelial growth factor (VEGF) 




Figure 3: Comparison of cf-miR-210 levels in healthy donor controls and disease-free melanoma patients' plasma samples. Melanoma patients $(n=130)$ had significantly higher cf-miR-210 level than healthy donors $(n=35$; male: $n=19$, female: $\left.n=16 ;{ }^{*} p<0.001\right)$.

(A) Patients with no disease recurrence $(n=46)$

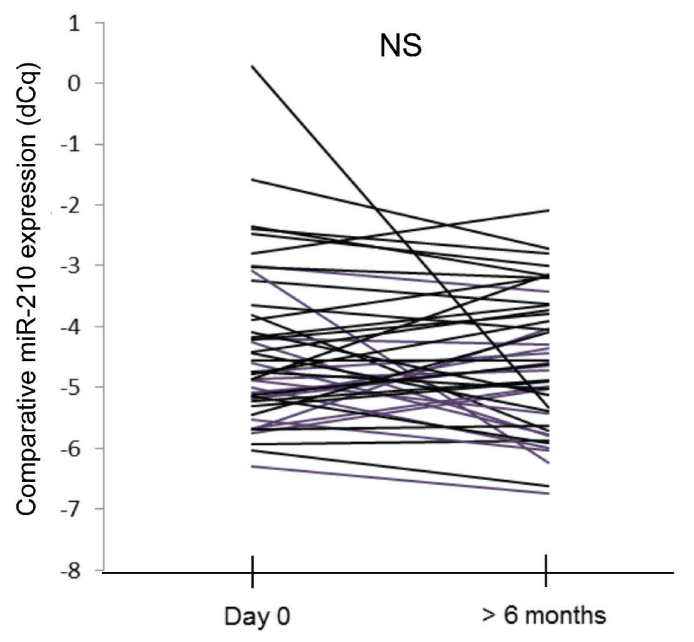

(B) Patients with disease recurrence $(n=42)$

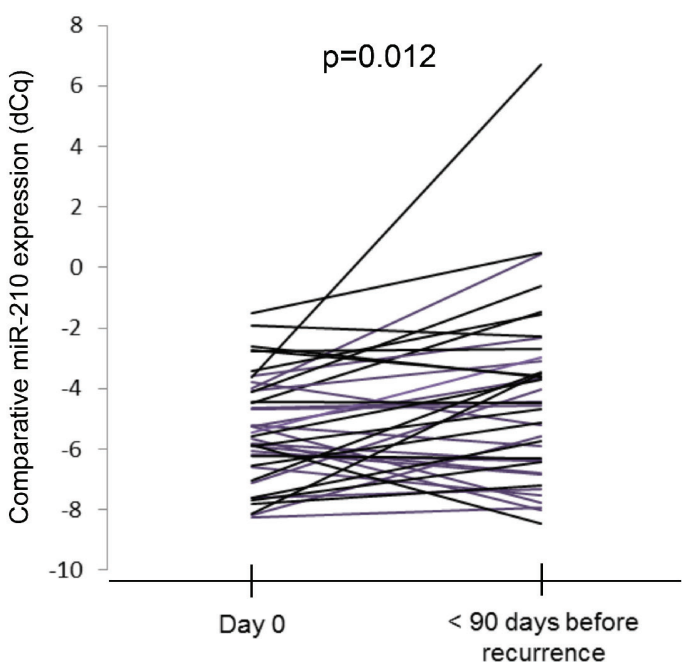

Figure 4: Comparison of cf-miR-210 levels in serial bleeds from the same patients. (A) Cf-miR-210 detection in patients without recurrence did not change significantly. (B) Patients who recurred experienced a significant increase in cf-miR-210 detection before recurrence $(p=0.012)$. 
(A)

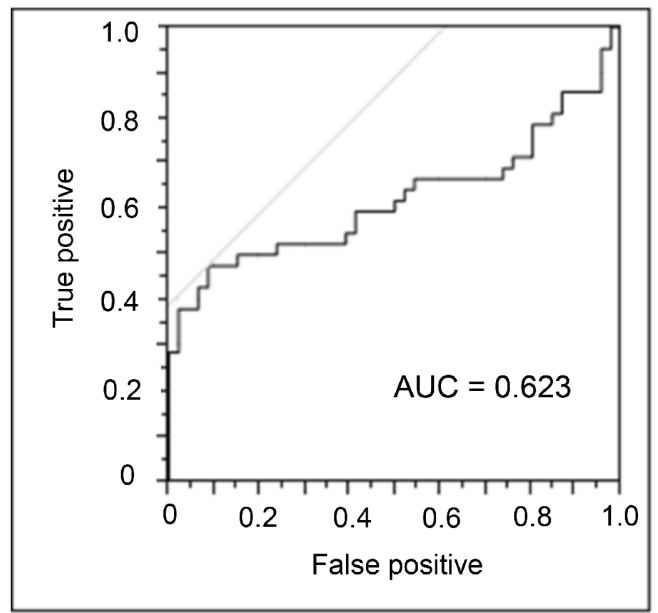

(B)


Figure 5: Increase in ef-miR-210 level correlated with recurrence and poor prognosis. (A) ROC analysis: Change in cfmiR-210 was a significant predictor of recurrence (cut off: one dCq difference; Sensitivity: $47.6 \%$, Specificity: $91.3 \%$. AUC $=0.623$; $p=0.015$ ). Positive: Recurrence $<2$ yrs, Negative: No recurrence $>5$ yrs. (B) Cf-miR-210 expression was significantly related to disease outcome (DFS, MSS: $p<0.001$ ). Comparison of AJCC stage III melanoma patients with either $>1 \mathrm{dCq}$ increase in cf-miR-210 levels from baseline to pre-treatment bleed ( $n=24$ patients) versus patients without such an increase ( $n=64$ patients). Blue line: No increase in cf-miR-210. Red line: Increase in cf-miR-210.

induction and accompanied angiogenesis and metastasis $[33,34]$. Metastasis develops through multiple events including angiogenesis [35], and miR-210 is also wellknown to induce angiogenesis after exposed to hypoxia [32]. Our finding could demonstrate hypoxia-inducible miR-210 has an important role during melanoma disease progression.

Cf-miR-210 level was elevated in the plasma of melanoma patients compared to healthy donor control plasma. These results suggested cf-miR-210 could be an indicator for systemic metastasis. In cohort A, there was no significant difference in cf-miR-210 expression between stage III and IV, this is likely because the patients had no presence of clinical disease at the time of blood draw. In addition, we demonstrated elevated cf-miR-210 was seen in the patients who subsequently developed systemic recurrence prior to their clinical diagnosis, and therefore could predict recurrence earlier.

In cohort B, we utilized the blood taken after the curative resection of melanoma as the baseline. Tumor derived cf-miRNA levels have been demonstrated to drop significantly seven days after surgery [36]. CfmiRNA expression level in our pretreatment plasma was not likely affected by the surgery itself, gender and age, or melanoma-derived from clinically evident disease because the bleeding point was at least 13 days after the definitive surgery. This implies that cf-miR-210 level may be higher in melanoma patients even after the curative resections compared to healthy donor control, possibly from clinically occult tumors. Considering this finding, it is quite important to follow the expression level serially to detect systemic progression of the disease. One of the major problems after surgical resection of melanoma tumors to render the patient clinically disease-free is the follow-up and identification of early systemic disease recurrence. With multiple FDA approved drugs, effective combinations of drugs, as well as many new phase II/III drugs in the pipeline, melanoma patients have many more opportunities of having extended survival than several years ago. Therefore, the earlier detection of disease recurrence is even more important; such that treatment can be assigned appropriately to result in prolong survival.

LDH levels were also analyzed in plasma taken on either the same day, or $<30$ days for which cf-miR-210 was assessed. LDH is currently the only approved biomarker routinely used in assessing for and level of 
(A)

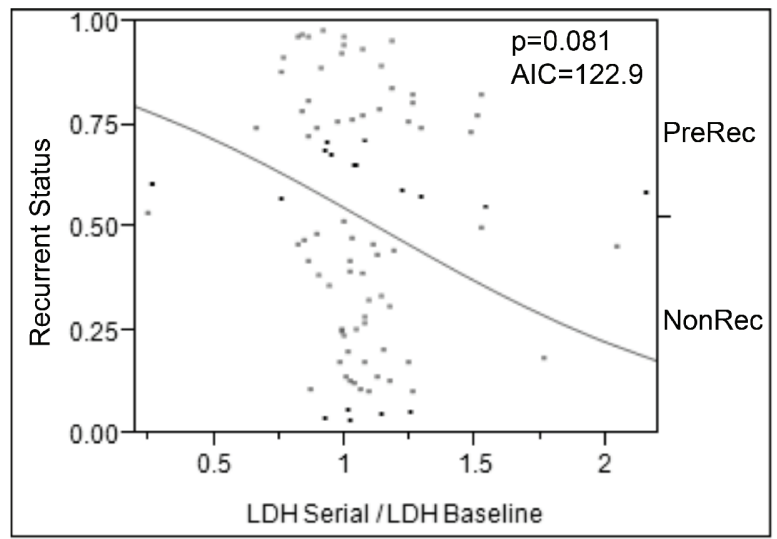

(B)

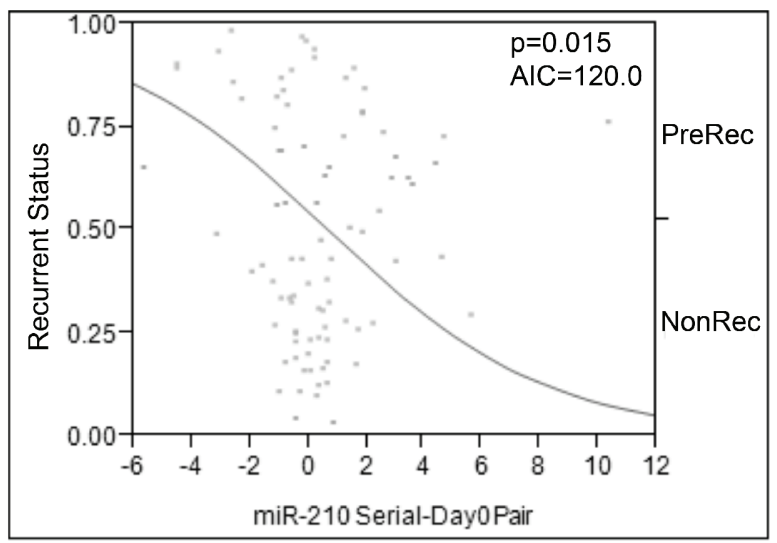

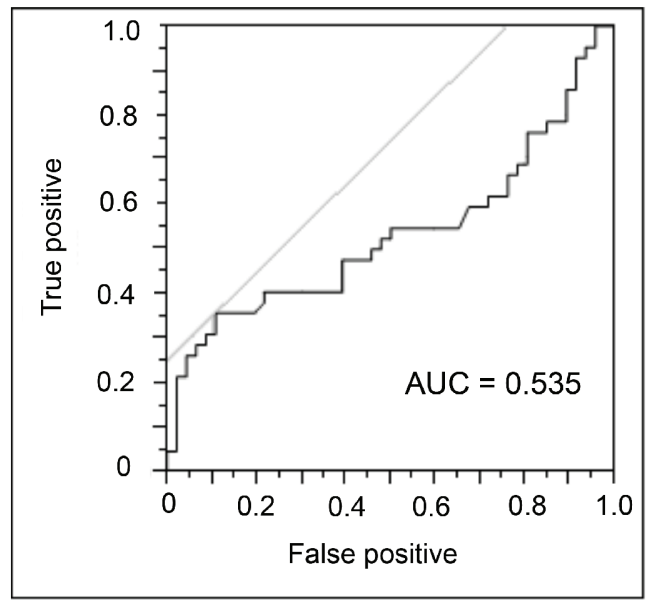

Figure 6: Comparison of change in baseline LDH vs change in ef-miR-210 dCq. (A) ROC analysis was performed to determine the cutoff for LDH changes (LDH Cutoff: 1.175-fold change from baseline; AUC $=0.535$ ). Change in LDH level did not predict recurrence $(p=0.810$, AIU $=122.91)(\mathrm{A})$, while change in cf-miR-210 was a significant predictor of recurrence $(\mathbf{B})(p=0.15$, AIU $=120.01)$. Positive: Recurrence $<2$ yrs, Negative: No recurrence $>5$ yrs.

metastasis in melanoma patients. Elevated level of LDH in serum is an independent and highly significant negative predictor of survival outcome among stage IV patients [1] even though it has been a second determinant of distant metastasis staging in melanoma $[37,38]$. In addition, it is rarely a singular indicator of disease recurrence and survival prediction for any other melanoma patients except for AJCC Stage IV patients [1]. In this study, cfmiR-210 was the only predictor of disease recurrence for Stage III melanoma patients, raising the possibility that cf-miR-210 could potentially be a novel biomarker for predicting disease recurrence or prognosis of these melanoma patients.

In conclusion, increasing cf-miR-210 in advanced melanoma patients is a prognostic biomarker of risk of recurrence and for disease outcome that may allow us to monitor patients better and begin treatment earlier. The RT-qPCR-DP is a sufficient assay to detect different levels of miRNA in small volumes of blood. The ability to detect and quantify cf-miR-210 may ultimately assist in clinical decision making such that it can be performed at multiple times to assess disease response to various treatments and aid in the earlier detection of systemic recurrence.

\section{MATERIALS AND METHODS}

\section{FFPE tissue analysis}

All FFPE tissue samples assessed were obtained from 108 patients at Saint John's Health Center (SJHC) between 1995 and 2010 (Supplemental Table 1). Tissue samples were fixed and embedded using the SJHC department of pathology's the Tissue-Tek ${ }^{\circledR}$ VIP ${ }^{\circledR} 6$ Processing for Histology protocol. The samples consisted of PRM $(n=21)$, LNM $(n=43)$, and DOM $(n=44)$. DOM sites were lung $(n=16)$, bowel $(n=12)$, liver $(n=6)$, distant skin $(n=3)$, brain $(n=2)$ and others $(n=5)$. All samples and clinical information were 
obtained in accordance with the SJHC/John Wayne Cancer Institutional (JWCI) Review Board (IRB) approval.

\section{Patients in the clinical trial and blood collection}

In the MMAIT, patients after the complete resection of AJCC stage III/IV melanoma were randomly assigned to the two treatment arms: BCG plus a Canvaxin ${ }^{\circledR}$ melanoma vaccine or BCG plus a placebo $[5,6]$. The clinical trials were registered as FDA phase III trials, with two trials for AJCC stage III and IV cutaneous melanoma between 1998 and $2005[5,6]$. All the clinical data for patients were prospectively collected after informed consent and under FDA guidelines. BCG with or without vaccine was given as an immune adjuvant as previously described [5]. The blood specimens were collected before adjuvant treatment and at several serial bleed defined points, under GLP conditions and during adjuvant treatment. Finally, the trial showed no clinical difference between the two randomized treatment arms.

Initially three different patients' plasma samples from AJCC stage III were used to assess whether RTqPCR-DP can detect cf-miRNA in plasma and show reproducibility. For the pilot study, plasma samples were obtained from six healthy donors and AJCC stage III $(n=20)$ and IV $(n=26)$ melanoma patients with disease present. For the verification study, plasma samples were obtained from healthy donors $(n=35)$, and 218 clinically well-annotated patients were selected, which comprised two patient cohorts (A: $n=130$; B: $n=88$ ), from the MMAIT. Patient cohort A contained 130 AJCC stage III $(n=60)$ and IV $(n=70)$ disease-free patients with specific bleed points (Supplemental Table 2A, 2B). The cohort was utilized for comparing cf-miR-210 expression level between disease-free melanoma patients and healthy donors. Cohort B contained 88 stage III patients, separated by those that either recurred $<2$ years after surgery $(n=46)$ or that did not recur for $>5$ years after surgery $(n=42$; Table 1). In cohort B, paired serial bleeds from the same patients were assessed beginning prior to adjuvant treatment, and either $<90$ days prior to recurrence for recurrent patients or $>6$ months after adjuvant treatment started for non-recurrent patients, to determine if cfmiR-210 detection changed prior to the recurrence. The pretreatment bleed points ranged from 13 to 95 days after the surgery.

This study was performed in concordance with the Reporting Recommendations for Tumor Marker Prognostic Studies (REMARK) of the National Cancer Institute [39].

\section{Blood specimens}

The clinical trial and companion blood studies were approved by the IRB at SJHC/JWCI and all other participating centers. All blood specimens were taken together under a specific standard operating procedure (SOP) approved by the MMAIT cohort for all sites and monitored accordingly for quality control. Blood processing was performed under SOP in GLP conditions predetermined prior to the start of the clinical trial. Peripheral blood was collected in $4.5 \mathrm{ml}$ blue-top tubes containing anticoagulant sodium citrate. Blood samples were centrifuged $(1,300 \times \mathrm{g}, 10 \mathrm{~min}$.) to separate plasma, plasma was carefully collected, and then immediately processed through a 13-mm filter to remove potential cell contamination and blood related debris. Plasma was then aliquoted and cryopreserved in liquid nitrogen under GLP conditions until used for this study.

\section{RNA extraction from FFPE tissue samples}

For RNA extraction, 10 sections at $10 \mu \mathrm{m}$ thick were cut from each FFPE tissue block. Deparaffinized tissues were digested with Proteinase K (Applied Biosystems) at $50^{\circ} \mathrm{C}$ for $3 \mathrm{hrs}$ as previously described [40]. Samples were homogenized and lysed in RNA-Solv Reagent (Omega Bio-Tek) followed by chloroform to denature proteins. Overnight precipitation was performed with isopropanol in addition to Pellet Paint NF (Novagen) as a carrier to pellet the RNA. Total RNA yield was determined and assessed for purity by using ultraviolet spectrophotometry and Quant-iT RiboGreen RNA assay kit (Life Technologies).

\section{Reverse transcriptional quantitative PCR (RT-qPCR)}

Reverse-transcription of $250 \mathrm{ng}$ of total RNA extracted from FFPE samples was performed using qScript cDNA Synthesis Kit (Quanta Biosciences). The transcribed cDNA was diluted 10-fold with nuclease-free water after the mixture was incubated at $37^{\circ} \mathrm{C}$ for $2 \mathrm{hrs}$.

Each qPCR (PerfeCTa ${ }^{\circledR}$ microRNA Assay; Quanta Biosciences) contained $4.6 \mu$ diluted cDNA, $5 \mu \mathrm{l}$ of $2 \mathrm{x}$ PerfeCTa SYBR Green SuperMix (Quanta Bioscience), $0.2 \mu \mathrm{lmiR}$-specific primer, and $0.2 \mu \mathrm{l}$ PCR Universal primer. Samples were assessed in triplicates at $95^{\circ} \mathrm{C}$ for 2 min: 40 cycles at $95^{\circ} \mathrm{C}$ for $5 \mathrm{sec}$ and $60^{\circ} \mathrm{C}$ for $30 \mathrm{sec}$. A CFX96 Touch $^{\mathrm{TM}}$ Real-Time PCR Detection System (BIORAD) was used for qPCR with melting-curve analysis. Each sample was assessed in triplicates with positive and negative cell lines, plasma controls and individual reagent controls to be used as inter-plate correction. The expression level of miRNA was recorded as $\mathrm{dCq}$ as follows: $\mathrm{dCq}=$ mean Cq values (1 ng of RNA from M14) mean $\mathrm{Cq}$ values (each sample).

\section{RT-qPCR-DP}

RT-qPCR-DP was performed with $10 \mu \mathrm{l}$ of plasma. Each plasma sample was mixed with $10 \mu \mathrm{l}$ of $2.5 \%$ Tween as previously described [18] to deactivate and solubilize 
Table 1: Patient characteristics

\begin{tabular}{|c|c|}
\hline Clinicopathological Factor & \# Patients (\%) \\
\hline \multicolumn{2}{|l|}{ Treatment Arm } \\
\hline Canvaxin $^{\circledR}$ & $36(41 \%)$ \\
\hline Recurrent (within 2 years) & $16(44 \%)$ \\
\hline Nonrecurrent $(>5$ years $)$ & $20(56 \%)$ \\
\hline Placebo & $52(59 \%)$ \\
\hline Recurrent (within 2 years) & $26(50 \%)$ \\
\hline Nonrecurrent ( $>5$ years) & $26(50 \%)$ \\
\hline \multicolumn{2}{|l|}{ Gender } \\
\hline Male & $56(64 \%)$ \\
\hline Female & $32(36 \%)$ \\
\hline Age $($ Mean \pm Std $)$ & $49.3 \pm 14.1$ \\
\hline \multicolumn{2}{|l|}{ Palpable LN Status } \\
\hline Palpable & $43(49 \%)$ \\
\hline Non-palpable & $45(51 \%)$ \\
\hline \multicolumn{2}{|l|}{ LN Positive } \\
\hline 1 positive & $45(51 \%)$ \\
\hline 2-3 positive & $24(27 \%)$ \\
\hline $4+$ positive & $19(22 \%)$ \\
\hline \multicolumn{2}{|l|}{ Primary Tumor Ulceration } \\
\hline Yes & $32(36 \%)$ \\
\hline No & $31(35 \%)$ \\
\hline Unknown & $25(28 \%)$ \\
\hline Maximum LDH (Mean \pm Std) & $318 \pm 202$ \\
\hline \multicolumn{2}{|c|}{ Primary Tumor Breslow Thickness } \\
\hline$\leq 1.00 \mathrm{~mm}$ & $16(18 \%)$ \\
\hline $1.01-2.00 \mathrm{~mm}$ & $18(20 \%)$ \\
\hline $2.01-4.00 \mathrm{~mm}$ & $22(25 \%)$ \\
\hline$>4.00 \mathrm{~mm}$ & $24(27 \%)$ \\
\hline Unknown & $8(9 \%)$ \\
\hline \multicolumn{2}{|l|}{ Primary Tumor Site } \\
\hline Head/Neck & $9(10 \%)$ \\
\hline Trunk & $39(44 \%)$ \\
\hline Extremity & $33(38 \%)$ \\
\hline Other & $3(3 \%)$ \\
\hline Unknown & $4(5 \%)$ \\
\hline
\end{tabular}


proteins that would affect the RT-qPCR results. In addition, it dissolves exosomes and lipid bound miRNA in the plasma. In the RT step, $20 \mu$ of RT reagent mixture containing $5 \mathrm{X}$ first strand buffer (PROMEGA), 10mmol/L deoxynucleoside-5' -triphoshate, miRNA-specific RT primer, RNasin, Moloney murine leukemia virus reverse transcriptase, and nuclease-free water was added to each plasma sample followed by a 2 hrs incubation at $37^{\circ} \mathrm{C}$ with a final $5 \mathrm{~min}$ enzyme inactivation at $95^{\circ} \mathrm{C}$. The transcribed cDNA was centrifuged at $9,000 \mathrm{~g}$ for $5 \mathrm{~min}$ to eliminate the protein precipitate, and then the supernatant was purified using Centri-Sep 8 well strips kit (Princeton Separations) according to the manufacturer's instruction. The Centri-Sep removes lipids, inhibitor proteins, RT and nucleic acid impurities, and aids in purification. It also eliminates free and labeled dNTPs from DNA or RNA. DNase treatment was not necessary for this assay as previously described [18].

Each qPCR reaction contained $4.5 \mu \mathrm{l}$ of cDNA, $5 \mu \mathrm{l}$ of PerfeCTa SYBR FastMix for iQ (Quanta Bioscience), $0.2 \mu \mathrm{l}$ of miRNA-specific forward primer, $0.2 \mu \mathrm{l}$ of universal reverse primer, and $0.1 \mu \mathrm{l}$ of nuclease-free water. Samples were assessed in triplicates in 40 cycles at $95^{\circ} \mathrm{C}$ for $10 \mathrm{sec}$ and of $42^{\circ} \mathrm{C}$ for $30 \mathrm{sec}$. Positive and negative control, in addition to reagent controls were implemented and used as inter-plate correction. Standard curves were generated by using five serially diluted (0.1-10 ng) M14 $\mathrm{Cq}$ values. The comparative quantification of miRNA expression level was recorded as the $\mathrm{dCq}$ as described above.

Due to the small nature of these miRNAs, conventional RT and RT-qPCR cannot be used to accurately detect miRNAs. A short unique RT-Specific primer is designed to target the 3' end of miRNAs while a universal tag is designed to the 5' end of the RT-Specific primer. The universal PCR reverse primer complements the universal tag that was added to the RT step. PCR universal reverse primer sequence: 5'-CATGATCAGCTGGGCCA-3'. The miR-210 primer sequence was: 5'-CATGATCAG CTGGGCCAAGATCAGCCG-3' (rt-specific); 5'-CTGTG CGTGT-3' (forward).

\section{Biostatistical analysis}

The expression levels of miRNAs were compared by using Wilcoxon Rank Sum test for individual bleed points and using the paired $t$-test for comparing serial bleeds within patients. Pearson product-moment correlation coefficient was used to analyze correlations between two groups. ROC curve was established to evaluate the diagnostic value of plasma miRNAs for the differentiation between melanoma recurrent patients and non-recurrent patients. The Fisher's exact test was used in the analysis of contingency tables. Univariate survival analysis was performed by applying the log-rank test to cf-miRNA expression levels and standard melanoma prognostic factors. A $p$-value of $<0.05$ was considered statistically significant. All analysis was performed with JMP 10 software (SAS Institute).

\section{ACKNOWLEDGMENTS}

This work was supported by the Dr. Miriam and Sheldon G. Adelson Medical Research Foundation, Ruth and Martin H. Weil Fund, National Institute of Health, and the National Cancer Institute (PO1 CA029605 Project II and Core C, and RO1 CA167967).

\section{CONFLICT OF INTEREST}

All the authors had no potential conflict of interest to disclose.

\section{Editorial note}

This paper has been accepted based in part on peerreview conducted by another journal and the authors' response and revisions as well as expedited peer-review in Oncotarget.

\section{Abbreviation}

AIC - Akaike information criterion, AJCC American Joint Committee on Cancer, AUC - area under the curve, BCG - Bacillus Calmette-Guerin, cf - cellfree, DFS - disease-free survival, FFPE - Formalin-fixed paraffin-embedded, IRB - Institutional review board, LDH - lactate dehydrogenase, miRNA - microRNA, MMAIT - Malignant Melanoma Active Immunotherapy Trial, MSS - melanoma specific survival, qPCR quantitative polymerase chain reaction, REMARK Reporting Recommendations for Tumor Marker Prognostic Studies, ROC - receiver-operating characteristics, RT reverse transcription, RT-qPCR-DP - RT-qPCR directly in plasma assay, SOP - standard operating procedure, 3' UTR 3 ' untranslated regions.

\section{REFERENCES}

1. Balch CM, Gershenwald JE, Soong SJ, Thompson JF, Atkins MB, Byrd DR, Buzaid AC, Cochran AJ, Coit DG, Ding S, Eggermont AM, Flaherty KT, Gimotty PA, Kirkwood JM, McMasters KM, Mihm MC Jr., et al. Final version of AJCC melanoma staging and classification. Journal of clinical oncology. 2009; 27:6199-6206.

2. Morton DL, Thompson JF, Cochran AJ, Mozzillo N, Nieweg OE, Roses DF, Hoekstra HJ, Karakousis CP, Puleo CA, Coventry BJ, Kashani-Sabet M, Smithers BM, Paul E, Kraybill WG, McKinnon JG, Wang HJ, et al. Final trial report of sentinel-node biopsy versus nodal observation in melanoma. The New England journal of medicine. 2014; 370:599-609. 
3. Robert C, Ribas A, Wolchok JD, Hodi FS, Hamid O, Kefford R, Weber JS, Joshua AM, Hwu WJ, Gangadhar TC, Patnaik A, Dronca R, Zarour H, Joseph RW, Boasberg P, Chmielowski B, et al. Anti-programmed-death-receptor-1 treatment with pembrolizumab in ipilimumab-refractory advanced melanoma: a randomised dose-comparison cohort of a phase 1 trial. Lancet. 2014; 384:1109-1117.

4. Larkin J, Ascierto PA, Dreno B, Atkinson V, Liszkay G, Maio M, Mandala M, Demidov L, Stroyakovskiy D, Thomas L, de la Cruz-Merino L, Dutriaux C, Garbe C, Sovak MA, Chang I, Choong N, et al. Combined Vemurafenib and Cobimetinib in BRAF-Mutated Melanoma. The New England journal of medicine. 2014; 371:1867-1876.

5. Hoshimoto S, Faries MB, Morton DL, Shingai T, Kuo C, Wang HJ, Elashoff R, Mozzillo N, Kelley MC, Thompson JF, Lee JE, Hoon DS. Assessment of prognostic circulating tumor cells in a phase III trial of adjuvant immunotherapy after complete resection of stage IV melanoma. Annals of surgery. 2012; 255:357-362.

6. Hoshimoto S, Shingai T, Morton DL, Kuo C, Faries MB, Chong K, Elashoff D, Wang HJ, Elashoff RM, Hoon DS. Association between circulating tumor cells and prognosis in patients with stage III melanoma with sentinel lymph node metastasis in a phase III international multicenter trial. Journal of clinical oncology. 2012; 30:3819-3826.

7. Ambros V. microRNAs: tiny regulators with great potential. Cell. 2001; 107:823-826.

8. Bartel DP. MicroRNAs: genomics, biogenesis, mechanism, and function. Cell. 2004; 116:281-297.

9. Kim VN. MicroRNA biogenesis: coordinated cropping and dicing. Nature reviews Molecular cell biology. 2005; 6:376-385.

10. Gantier MP, McCoy CE, Rusinova I, Saulep D, Wang D, $\mathrm{Xu}$ D, Irving AT, Behlke MA, Hertzog PJ, Mackay F, Williams BR. Analysis of microRNA turnover in mammalian cells following Dicer1 ablation. Nucleic acids research. 2011; 39:5692-5703.

11. Mitchell PS, Parkin RK, Kroh EM, Fritz BR, Wyman SK, Pogosova-Agadjanyan EL, Peterson A, Noteboom J, O'Briant KC, Allen A, Lin DW, Urban N, Drescher CW, Knudsen BS, Stirewalt DL, Gentleman R, et al. Circulating microRNAs as stable blood-based markers for cancer detection. Proceedings of the National Academy of Sciences of the United States of America. 2008; 105:10513-10518.

12. Schwarzenbach H, Nishida N, Calin GA, Pantel K. Clinical relevance of circulating cell-free microRNAs in cancer. Nature reviews Clinical oncology. 2014; 11:145-156.

13. Sourvinou IS, Markou A, Lianidou ES. Quantification of circulating miRNAs in plasma: effect of preanalytical and analytical parameters on their isolation and stability. The Journal of molecular diagnostics: JMD. 2013; 15:827-834.

14. Friedman EB, Shang S, de Miera EV, Fog JU, Teilum MW, Ma MW, Berman RS, Shapiro RL, Pavlick AC, Hernando E, Baker A, Shao Y, Osman I. Serum microRNAs as biomarkers for recurrence in melanoma. Journal of translational medicine. 2012; 10:155.

15. Shiiyama R, Fukushima S, Jinnin M, Yamashita J, Miyashita A, Nakahara S, Kogi A, Aoi J, Masuguchi S, Inoue $\mathrm{Y}$, Ihn H. Sensitive detection of melanoma metastasis using circulating microRNA expression profiles. Melanoma research. 2013; 23:366-372.

16. Umetani N, Giuliano AE, Hiramatsu SH, Amersi F, Nakagawa T, Martino S, Hoon DS. Prediction of breast tumor progression by integrity of free circulating DNA in serum. Journal of clinical oncology. 2006; 24:4270-4276.

17. Umetani N, Kim J, Hiramatsu S, Reber HA, Hines OJ, Bilchik AJ, Hoon DS. Increased integrity of free circulating DNA in sera of patients with colorectal or periampullary cancer: direct quantitative PCR for ALU repeats. Clinical chemistry. 2006; 52:1062-1069.

18. Asaga S, Kuo C, Nguyen T, Terpenning M, Giuliano AE, Hoon DS. Direct serum assay for microRNA-21 concentrations in early and advanced breast cancer. Clinical chemistry. 2011; 57:84-91.

19. Covello KL, Simon MC, Keith B. Targeted replacement of hypoxia-inducible factor-1alpha by a hypoxia-inducible factor-2alpha knock-in allele promotes tumor growth. Cancer research. 2005; 65:2277-2286.

20. Gee HE, Ivan C, Calin GA, Ivan M. HypoxamiRs and Cancer: From Biology to Targeted Therapy. Antioxidants \& redox signaling. 2014; 21:1220-1238.

21. Camps C, Buffa FM, Colella S, Moore J, Sotiriou C, Sheldon H, Harris AL, Gleadle JM, Ragoussis J. hsa-miR-210 Is induced by hypoxia and is an independent prognostic factor in breast cancer. Clinical cancer research. 2008; 14:1340-1348.

22. Gee HE, Camps C, Buffa FM, Patiar S, Winter SC, Betts G, Homer J, Corbridge R, Cox G, West CM, Ragoussis J, Harris AL. hsa-mir-210 is a marker of tumor hypoxia and a prognostic factor in head and neck cancer. Cancer. 2010; 116:2148-2158.

23. Huang X, Ding L, Bennewith KL, Tong RT, Welford SM, Ang KK, Story M, Le QT, Giaccia AJ. Hypoxia-inducible mir-210 regulates normoxic gene expression involved in tumor initiation. Molecular cell. 2009; 35:856-867.

24. Wang J, Zhao J, Shi M, Ding Y, Sun H, Yuan F, Zou Z. Elevated expression of miR-210 predicts poor survival of cancer patients: a systematic review and meta-analysis. PloS one. 2014; 9:e89223.

25. Kim HW, Haider HK, Jiang S, Ashraf M. Ischemic preconditioning augments survival of stem cells via miR-210 expression by targeting caspase- 8 -associated protein 2 . The Journal of biological chemistry. 2009; 284:33161-33168.

26. Crosby ME, Kulshreshtha R, Ivan M, Glazer PM. MicroRNA regulation of DNA repair gene expression in hypoxic stress. Cancer research. 2009; 69:1221-1229.

27. Caramuta S, Egyhazi S, Rodolfo M, Witten D, Hansson J, Larsson C, Lui WO. MicroRNA expression profiles 
associated with mutational status and survival in malignant melanoma. The Journal of investigative dermatology. 2010; 130:2062-2070.

28. Mueller DW, Rehli M, Bosserhoff AK. miRNA expression profiling in melanocytes and melanoma cell lines reveals miRNAs associated with formation and progression of malignant melanoma. The Journal of investigative dermatology. 2009; 129:1740-1751.

29. Philippidou D, Schmitt M, Moser D, Margue C, Nazarov PV, Muller A, Vallar L, Nashan D, Behrmann I, Kreis S. Signatures of microRNAs and selected microRNA target genes in human melanoma. Cancer research. 2010; 70:4163-4173.

30. Segura MF, Belitskaya-Levy I, Rose AE, Zakrzewski J, Gaziel A, Hanniford D, Darvishian F, Berman RS, Shapiro RL, Pavlick AC, Osman I, Hernando E. Melanoma MicroRNA signature predicts post-recurrence survival. Clinical cancer research: an official journal of the American Association for Cancer Research. 2010; 16:1577-1586.

31. Leidinger P, Keller A, Borries A, Reichrath J, Rass K, Jager SU, Lenhof HP, Meese E. High-throughput miRNA profiling of human melanoma blood samples. BMC cancer. 2010; 10:262.

32. Hu S, Huang M, Li Z, Jia F, Ghosh Z, Lijkwan MA, Fasanaro P, Sun N, Wang X, Martelli F, Robbins RC, Wu JC. MicroRNA-210 as a novel therapy for treatment of ischemic heart disease. Circulation. 2010; 122:S124-131.

33. Welsh SJ, Bellamy WT, Briehl MM, Powis G. The redox protein thioredoxin-1 (Trx-1) increases hypoxia-inducible factor 1alpha protein expression: Trx-1 overexpression results in increased vascular endothelial growth factor production and enhanced tumor angiogenesis. Cancer research. 2002; 62:5089-5095.
34. Raleigh JA, Calkins-Adams DP, Rinker LH, Ballenger CA, Weissler MC, Fowler WC Jr., Novotny DB, Varia MA. Hypoxia and vascular endothelial growth factor expression in human squamous cell carcinomas using pimonidazole as a hypoxia marker. Cancer research. 1998; 58:3765-3768.

35. Malanchi I, Santamaria-Martinez A, Susanto E, Peng H, Lehr HA, Delaloye JF, Huelsken J. Interactions between cancer stem cells and their niche govern metastatic colonization. Nature. 2012; 481:85-89.

36. Zhao A, Li G, Peoc'h M, Genin C, Gigante M. Serum miR-210 as a novel biomarker for molecular diagnosis of clear cell renal cell carcinoma. Experimental and molecular pathology. 2013; 94:115-120.

37. Deichmann M, Benner A, Bock M, Jackel A, Uhl K, Waldmann V, Naher H. S100-Beta, melanoma-inhibiting activity, and lactate dehydrogenase discriminate progressive from nonprogressive American Joint Committee on Cancer stage IV melanoma. Journal of clinical oncology. 1999; 17:1891-1896.

38. Tarhini AA, Stuckert J, Lee S, Sander C, Kirkwood JM. Prognostic significance of serum S100B protein in highrisk surgically resected melanoma patients participating in Intergroup Trial ECOG 1694. Journal of clinical oncology. 2009; 27:38-44.

39. McShane LM, Altman DG, Sauerbrei W, Taube SE, Gion M, Clark GM. Reporting recommendations for tumor marker prognostic studies (REMARK). Journal of the National Cancer Institute. 2005; 97:1180-1184.

40. Arigami T, Narita N, Mizuno R, Nguyen L, Ye X, Chung A, Giuliano AE, Hoon DS. B7-h3 ligand expression by primary breast cancer and associated with regional nodal metastasis. Annals of surgery. 2010; 252:1044-1051. 